\title{
Special Series Guest Editorial: EUV Masks
}

\author{
Martin Burkhardt ${ }^{\mathrm{a}}$ and Vicky Philipsen ${ }^{\mathrm{b}}$ \\ ${ }^{a}$ IBM Research, Yorktown Heights, New York, United States \\ ${ }^{\mathrm{b}}$ IMEC, Leuven, Belgium
}

Our community is proud that EUV lithography has been implemented in high-volume manufacturing (HVM) for several years now. The current lithography infrastructure that uses an NA of 0.33 performs well with the existing EUV mask infrastructure, and the industry is anticipating the launch of new high NA tools. This continuous push of the resolution capability of these tools calls for continuous research to improve imaging. Aside from the EUV scanner, the mask is key to reaching the full imaging potential of EUV lithography. Therefore, mask technology is currently a very active area of research, where the mask materials and concepts are being challenged, engineered, examined, and matured to support next-generation lithography. For this reason, the publication of papers on this topic, in the form of a special series, felt appropriate at this time of transition from low NA to high NA tools.

We can divide the contributions of this section roughly into three categories:

- First, the papers focusing on considerations for conventional masks. Melvin and Jonckheere study the contribution of mask defectivity in stochastics of wafer printing, Rook et al. write about the reflectivity optimization of ion-beam deposited Mo/Si layers, and finally Heil et al. investigate EUV mask repair using an AIMS tool in conjunction with e-beam mask repair.

- Second, we have papers on pellicles. They all deal with carbon nanotube (CNT) pellicles, which were previously shown to have good properties in imaging and mechanics. While we still don't know if CNT pellicles will be used in HVM, most of the techniques developed in those papers should be universally applicable to any pellicles. This series consists of papers by Timmermans et al. on CNT pellicle tunability, by Pollentier et al. on scattering light metrology on pellicles, and finally by Bekaert et al. on first wafer imaging results using masks with CNT pellicles.

- Third, there are seven papers covering the topic of novel mask materials in different aspects. Two papers investigate the novel absorber masks for high-NA EUV lithography from the simulation perspective, Erdmann et al. identify the optima in the material solution space and Mesilhy et al. explain the diffraction phenomenon in low-n masks as a waveguide problem. From the experimental perspective, Wu et al. report on the characterization of novel absorber materials for high NA EUV, while Van Lare et al. discuss the first printing results at NA 0.33 of a low-n absorber mask. Furthermore, Burov et al. compare the simulated stochastic side lobe printing at NA 0.33 for different mask approaches. Ban et al. report on the understanding of mask thermal deformation due to exposures by simulations. Finally, Sherwin et al. investigate mask phase measurements using reflectivity measurements and show experimental confirmation of contamination deposits.

This journal has had special sections on Photomasks for EUV Lithography (2013, edited by C. Progler and F. Abboud) and Photomask Manufacturing Technology (2016, edited by M. Shibuya, M. Hoga, and K. Takehisa), which contained a lot of material on EUV masks. Following these special sections from the pre-HVM days of EUV, we now have a special series in the early HVM days, and if we assume a mean interval of four years between such special sections, we look forward to a special series on EUV masks in the mature HVM days and early high-NA HVM days, sometime around the year 2025.

In summary, this special series on EUV masks contains an up-to-date anthology of fresh, insightful, and high-end research papers for you to replenish your scientific thirst.

(C) 2021 Society of Photo-Optical Instrumentation Engineers (SPIE) 
This collection of papers would not have been possible without the effort of all authors and reviewers, and we are grateful for their time and dedication. Moreover, we appreciate the professional guidance of the JM3 editorial team with their unceasing enthusiasm. Finally, we are thankful for the advice and thrust of JM3 Editor-in-Chief Harry Levinson. We are very pleased with the result and hope you will also enjoy it. 